\title{
A Novel Circular RNA circPTCD3 Promotes Breast Cancer Progression Through Sponging miR-198
}

\author{
Zhaohui Zhang' \\ $\mathrm{HaO} \mathrm{Hu}^{2}$ \\ Qian $\mathrm{Li}^{\mathbf{1}}$ \\ Fumei $\mathbf{Y i}^{\mathbf{I}}$ \\ Yan'e Liu' \\ 'Department of Tumor Chemotherapy \\ and Radiation Sickness, Peking University \\ Third Hospital, Beijing, I0019I, People's \\ Republic of China; ${ }^{2}$ Beijing I00biotech \\ Co., Ltd., Beijing, 100006, People's \\ Republic of China
}

\begin{abstract}
Introduction: Circular RNAs (circRNAs), a new type of non-coding RNA, have been demonstrated to play critical roles in the progression of various of malignant cancers. However, the function of circRNAs in breast cancer is not clearly understood.

Methods: qPCR was used to evaluate the gene expression. Function studies including MTT, transwell, wound healing and colony formation assay were performed to evaluate the function of circPTCD3 in breast cancer. Luciferase and RNA pull-down assays were used to verify the interaction between circPTCD3 and miR-198. The xenograft model was established to evaluate the function of circPTCD3 in vivo.

Results: In the present study, we identified a novel circRNA termed as circPTCD3 which was indicated to be significantly up-regulated in breast cancer tissues and cell lines. The results revealed that ectopic expression of circPTCD3 promoted the cell proliferation, migration and colony formation ability of breast cancer cells. Constantly, silencing of circPTCD3 inhibited those of breast cancer cells. Furthermore, we identified that circPTCD3 was able to target miR-198 in breast cancer cell. miR-198 has the function of inhibiting proliferation and migration of breast cancer cells which can be reversed by circPTCD3.

Conclusion: Taken together, our findings for the first time identified a novel circRNA (circPTCD3) and revealed its oncogenic role in breast cancer. Mechanically, we reported that circPTCD3 served as a competing endogenous RNA (ceRNA) to sponge miR-198. These findings provide insights into breast cancer progression and also potential new targets for diagnosis or treatment of breast cancer.
\end{abstract}

Keywords: circPTCD3, breast cancer, miR-198

\section{Introduction}

Breast cancer (BC) is counted among the key causes leading to cancer death across the globe, and is named as one of the most frequently found cancers in women worldwide. ${ }^{1}$ Despite considerable advances in both the therapy and diagnosis of breast cancer, no effective treatment has been developed for such a type of hormone-insensitive cancer. ${ }^{2,3}$ The currently available treatment for breast cancer is considered challenging even now. Accordingly, the development of innovative drugs for treating $\mathrm{BC}$ with high specificity and effect is required.

Circular RNAs (circRNAs) are an innovative class of the noncoding RNAs, characterized by a covalently closed loop without 5'-3' polyadenylated tails, which are developed by backsplicing events. ${ }^{4}$ Currently, circRNAs have been illustrated as
Department of Tumor Chemotherapy and Radiation Sickness, Peking University Third Hospital, Huayuan Bei Road,

Haidian District, Beijing, I00I9I, People's

Republic of China

Email zhaobj429@sina.com 
highly conserved and abundant with high stability across various species. ${ }^{5}$ With numerous investigations underway, circRNA was observed as differentially expressed in various of cancers, which include prostate cancer, breast cancer and hepatocellular carcinoma. ${ }^{6-8}$ Also, they were indicated as having involvement in a number of physiological and pathophysiological processes including cell proliferation, invasion, apoptosis and differentiation.

MicroRNAs (miRNAs) are small endogenous noncoding RNAs capable of regulating the cell process by means of binding to the specific target genes. ${ }^{9}$ This binding has the potential to inhibit translation or induce the degradation of target mRNAs. CircRNAs primarily sponge miRs to exert an impact on not only the gene expression but also biological functions. ${ }^{10}$

In the current research work, we figured out an upregulated circular RNA hsa_circ_0055478 (circPTCD3) in both breast cancer tissues and cell lines. The loss and gain of function studies were carried out for the determination of the effect of circPTCD3 on tumorigenesis of breast cancer. Mechanistically, we predicted and verified the target miRNA of circPTCD3, which is miR-198. To summarize, circ-PTCD3 works as an oncogene in breast cancer, besides being likely to constitute a potential therapeutic target for breast cancer.

\section{Methods and Materials}

\section{Patients and Specimens}

Twenty-four breast cancer tumor tissue samples and adjacent normal tissues were collected from Peking University Third Hospital. The tissues were frozen in liquid nitrogen immediately after excision and then stored in $-80^{\circ} \mathrm{C}$. All experimental procedures were approved by the Ethics Committee of Peking University Third Hospital and in accordance with the Declaration of Helsinki of the World Medical Association. The written informed consent was obtained from each patient.

\section{Cell Culture}

The normal breast cell line MCF-10a and breast cancer cell lines (MCF-7, BT474, MDA-MB-231, SKBr-3) were purchased from Chinese Academy of Sciences. The culturing of cells was carried out in RPMI-1640 medium supplemented with $10 \%$ fetal bovine serum (FBS, Hyclone, USA). Each cell line was cultured at $37^{\circ} \mathrm{C}$ with $5 \% \mathrm{CO} 2$. In addition, the cells were treated with the indicated concentration, whereas the control cells received an equivalent amount of DMSO. The final concentration of DMSO is below $0.1 \%$.

\section{Cell Transfection}

miR-198 mimics and mimic control were synthesized by GenePharma (Shanghai, China). CircPTCD3 overexpressing vector (PLO-ciR/circPTCD3), siRNA for circPTCD3 (si-circPTCD3) and short hairpin RNA (shRNA) targeting circPTCD3 (sh-circPTCD3) were purchased from Ribobio (Guangzhou, China). Cells were seeded on six-well plates at the density of $8 \times 10^{5}$, followed by getting cultured in RPMI-1640 without FBS at $37^{\circ} \mathrm{C}$ for $12 \mathrm{~h}$ prior to the transfection. Cell transfection and co-transfection were carried out with the use of Lipofectamine 2000 (Invitrogen, CA, USA) in accordance with the manufacturer's instructions. $6 \mathrm{~h}$ following the transfection, the cell culture medium was replaced with RPMI-1640 medium, which was supplemented with $10 \%$ FBS. Cell lines stably expressing circPTCD3 or sh-circPTCD3 were established by selecting with G418 for 6 weeks.

\section{MTT}

Twenty-four hours following the transfection, cells were collected and resuspended in culture medium, followed by seeding on 96-well plates at a density of $1 \times 10^{4}$ cells/well. Moreover, $10 \mu \mathrm{L}$ CCK-8 solution was added to each well and incubated for another $2 \mathrm{~h}$ at $37^{\circ} \mathrm{C}$. Absorbance at 490 $\mathrm{nm}$ was detected using a microplate Reader (Biorad, CA, USA). Each sample was performed for at least 3 times.

\section{Transwell Assay}

Subsequent to the transfection, $1 \times 10^{5}$ of cells suspended in $200 \mu \mathrm{L}$ RPMI-1640 medium were seeded onto the upper transwell chamber (Corning, NY, USA). Following 24 hours of incubation at $37^{\circ} \mathrm{C}$, the cells on the surfaces of lower chambers were fixed using $20 \%$ methanol followed by staining with $1 \%$ crystal violet (Bicobio, Tianjin, China). Eventually, the cell colonies were both photographed and counted.

\section{Wound Healing Assay}

Subsequent to the transfection, the cells were seeded onto six-well plates followed by culturing in serum free medium for a period of 24 hours. Thereafter, cell monolayers were wounded using a $10 \mu \mathrm{L}$ pipette tip. After that, cells were replaced with fresh medium and cultured at $37^{\circ} \mathrm{C}$. The pictures were taken 48 after the wounding for the purpose of determining the wound-closing procedure. 


\section{Colony Formation Assay}

Following the transfection, the cells were seeded in 12well plates at the density of $100 /$ well. Subsequent to the incubation for 2 weeks, cells were fixed in $10 \%$ formaldehyde, together with staining with $1 \%$ crystal violet (Beyotime, Shanghai, China). Images were photographed under a microscope (Leica, Germany), besides counting the colonies that contained more than 50 cells.

\section{Xenograft Model}

All the animal experimental procedures were approved by the Ethics Committee of Peking University Third Hospital. All animal procedures were performed in accordance with institutional guidelines and approved by the Animal Studies Committee of Peking University Third Hospital. MCF-7 cells were harvested and resuspended in RPMI1640 medium. The nude mice were injected with a total number of $3 \times 10^{6} / 100 \mu \mathrm{L}$ cells subcutaneously at the posterior flank. In addition, tumour size was monitored through the measurement of the length (L) and width (W) using callipers every 3 days. Following a period of 28 days, the tumours were excised out from the sacrificed mice and weighed.

\section{PCR}

Total RNA was extracted with the use of TRIzol reagent (Invitrogen, CA, USA) in accordance with the manufacturer's instructions. Afterwards, RNA was reverse transcribed to cDNA with the help of a PrimeScript RT Reagent kit (Takara, Dalian, China). The real-time qPCR analysis was carried out with the help of a SYBR-Green PCR Master Mix (Thermo Fisher, MA, USA) in a 7900 PCR System (ABI, CA, USA). The use of GAPDH and U6 was made as internal controls for the mRNA and miRNA analysis, correspondingly. The relative expression levels were assessed with the use of the $2-\Delta \Delta \mathrm{Ct}$ methodology. All of the reactions were carried out in triplicate.

\section{Fluorescence in situ Hybridization (FISH)}

Alexa Fluor 555-labeled circPTCD3 probes were designed and synthesized by RiboBio (Guangzhou, China). FISH experiment was carried out using a fluorescent in Situ Hybridization Kit (RiboBio, Guangzhou, China). Then, $1 \times 10^{5}$ cells were seeded onto the slides and cultured for a period of $24 \mathrm{~h}$. Subsequent to fixing with $4 \%$ paraformaldehyde for $20 \mathrm{~min}$, followed by the permeabilization with $0.5 \%$ Triton X-100 for $10 \mathrm{~min}$, the cells were cultured at $37^{\circ} \mathrm{C}$ overnight. Eventually, the incubation of slides was carried out with DAPI for the purpose of staining the cell nucleus and observed under a fluorescence microscope (Leica, Wetzlar, Germany).

\section{RNA Pull Down}

The biotin-labelled circPTCD3 and miR-198 probe and their control probes were synthesized by Sangon Biotech (Shanghai, China). In addition, the probe-coated beads were generated through the co-incubation of the probe with the streptavidin-coated beads (Invitrogen, CA, USA) at $25^{\circ} \mathrm{C}$ for $2 \mathrm{~h}$. Cells were gathered and incubated with circPTCD3 probes overnight at $4^{\circ} \mathrm{C}$. Following that, the beads were eluted and the complex was purified with TRIzol (Takara, Dalian, China). Afterwards, the abundance of both circPTCD3 and miR-198 was analyzed by the qPCR.

\section{$\mathrm{IHC}$}

The tumour tissues were fixed in $4 \%$ paraformaldehyde for $24 \mathrm{~h}$, followed by dehydrating in a graded alcohol series and embedding in paraffin. The tissues were cut into $5 \mu \mathrm{m}$ sections. The sections were deparaffinised, rehydrated with a graded alcohol series and then incubated in $96^{\circ} \mathrm{C}$ with $0.01 \mathrm{~mol} / \mathrm{L}$ sodium citrate buffer for the antigen retrieval. Following the incubation in $5 \% \mathrm{H}_{2} \mathrm{O}_{2}$ for a period of $2 \mathrm{~h}$, the sections were incubated using anti-ki67 antibody overnight at $4^{\circ} \mathrm{C}$. Immunostaining was carried out with the use of streptavidin-peroxidase and diaminobenzidine (DAB) following the manufacturer's instructions (Beyotime, Shanghai, China). Eventually, the sections were not only observed under a fluorescence microscope (Leica, Wetzlar, Germany) but also imaged.

\section{Luciferase Reporter Assay}

Cells were co-transfected with miR-198 mimics or mimic control along with pGL3 reporter vectors (Promega, CA, USA) that contain the wild-type (Wt) or mutated (mut) binding sequence of circPTCD3. Forty-eight hours following the transfection, cells were gathered and analyzed with a Dual-Luciferase Reporter Assay kit (Promega, CA, USA). Luciferase activity was detected through the use of a GloMax fluorescence reader (Promega, CA, USA). Renilla luciferase activities were used as an internal control. Each of the assays was carried out in a minimum of triplicate.

\section{Statistics Analysis}

The results are expressed as mean $\pm \mathrm{SD}$. Statistical analyses were performed using SPSS 19.0 software (IBM, 
NY, USA). Student's $t$-test or one-way ANOVA were used to assess the statistical significance. Pearson analysis was carried out to assess the correlation. $\mathrm{P}<0.05$ and was considered statistically significant.

\section{Results}

\section{The Expression of circPTCD3 Was}

\section{Elevated in Breast Cancer Tissues and}

\section{Cells}

To explore the role of circPTCD3 in the progression of breast cancer, we first figured out the mechanism of ring formation. In accordance with the UCSC Genome Browser Home, circPTCD3 is produced at the PTCD3 gene locus, which contains exons 3-9. Subsequent to that, the head-totail splicing junction of has_circPTCD3 was verified by means of the Sanger sequencing, as presented in Figure 1A. Breast cancer cells were treated with Actinomycin $\mathrm{D}$ in order to inhibit the transcription after which the halflife of circPTCD3 was assessed. The results shed light on a longer half-life of circPTCD3 as compared with that of linear PTCD3 (Figure 1B). Besides that, a specific probe was designed and made to carry out the FISH experiment. Figure $1 \mathrm{C}$ demonstrates that circPTCD3 located primarily in cytoplasm. As we know, circRNAs have much more stability for their looped structure. Accordingly, for the purpose of confirming the stability of circPTCD3, RNase $\mathrm{R}$ was employed in the experiments. As the results suggested, following the RNase $\mathrm{R}$ treatment, the linear forms of PTCD3 showed an evident decline while no significant change was observed in the level of circPTCD3, indicating the high stability of circPTCD3 (Figure 1D). Then, we detected the expression level of circPTCD3 with the use of qPCR. The results illustrated that circPTCD3 was remarkably up-regulated in the breast cancer tissues and cells (Figure 1E and F).

\section{CircPTCD3 Promoted the Proliferation and Migration of Breast Cancer Cells}

Due to the upregulated expression in breast cancer tissues and cell lines, we speculated that circPTCD3 plays quite a crucial role in the progression of breast cancer. The gain and loss of function studies were carried out with the use of overexpressing vector (PLO-ciR/circPTCD3) and siRNA for circPTCD3 (si-circPTCD3). The qPCR analysis verified the overexpression or knockdown efficiency of the PLO-ciR /circPTCD3or si-circPTCD3, correspondingly (Figure 2A).
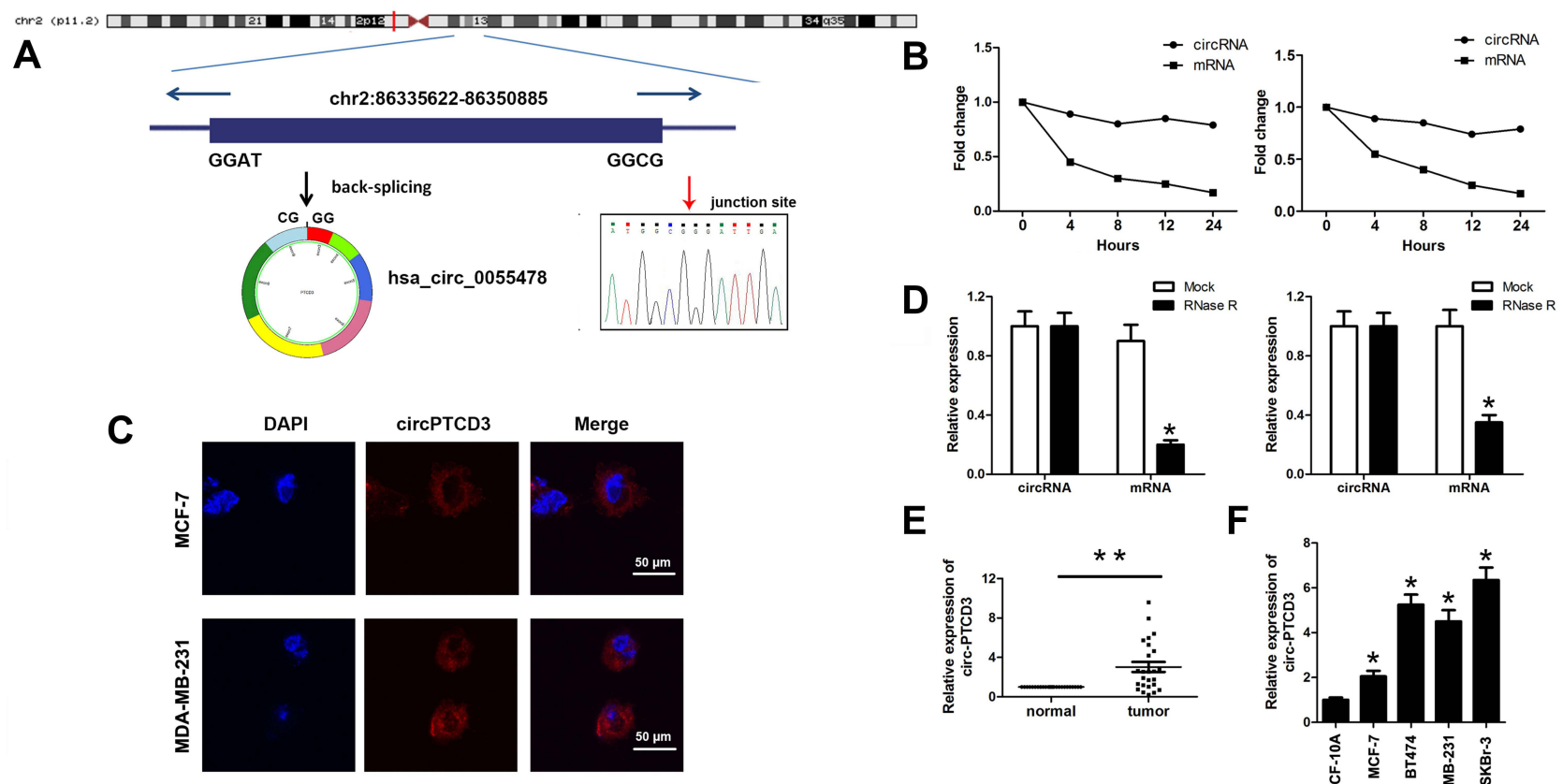

E

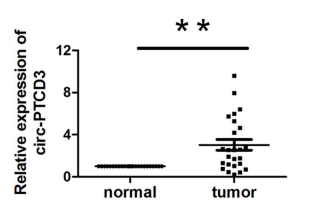

$\mathbf{F}$

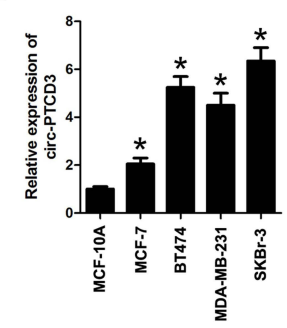

Figure I Hsa_circ_0055478 (circPTCD3) is upregulated in breast cancer specimens and cell lines. (A) Hsa_circ_0055478 also named as circPTCD3 was back-spliced by exons 3-9 of PTCD3 gene as the black arrow showed. The existence of circPTCD3 were confirmed by sanger sequencing, the red arrow shows the head-to-tail splicing junction site of circPTCD3. (B) After treatment with Actinomycin D, the amount of circular and linear PTCD3 from breast cancer cells were evaluated using qPCR. (C) FISH assay with specific probe was performed to detect the expression and location of circPTCD3. (D) After RNase R treatment, the expression of linear and circular PTCD3 were detected using qPCR. Expression level of circPTCD3 was evaluated by qPCR in breast cancer tissues (E) and cell lines (F) compare to the adjacent normal tissues and MCF-IOA cell. $n=6, * P<0.05$, compared with the normal, mock, MCF-IOA group correspondingly, $* * P<0.01$, compared with the normal. 
A

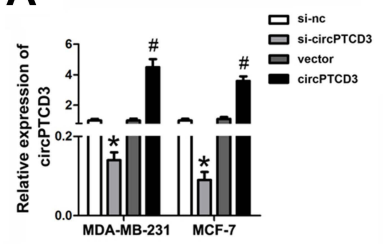

C

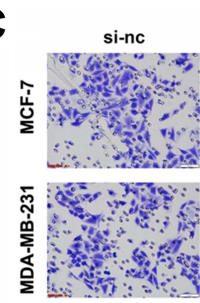

E
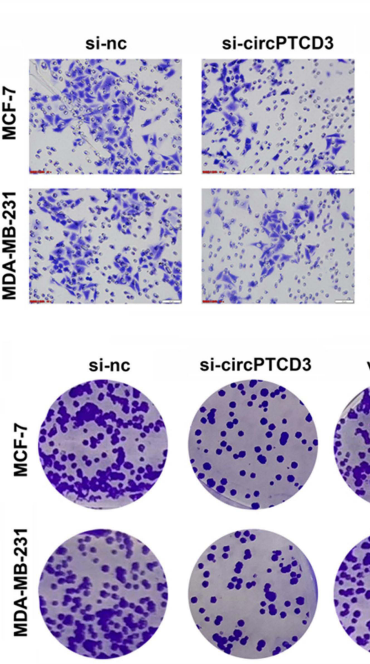

B

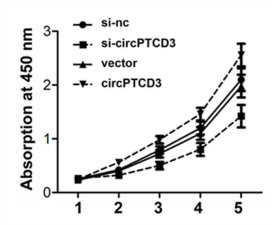

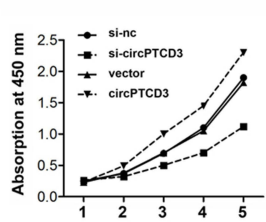

sinc
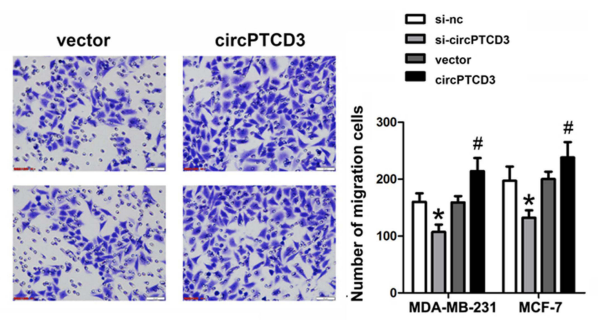

D

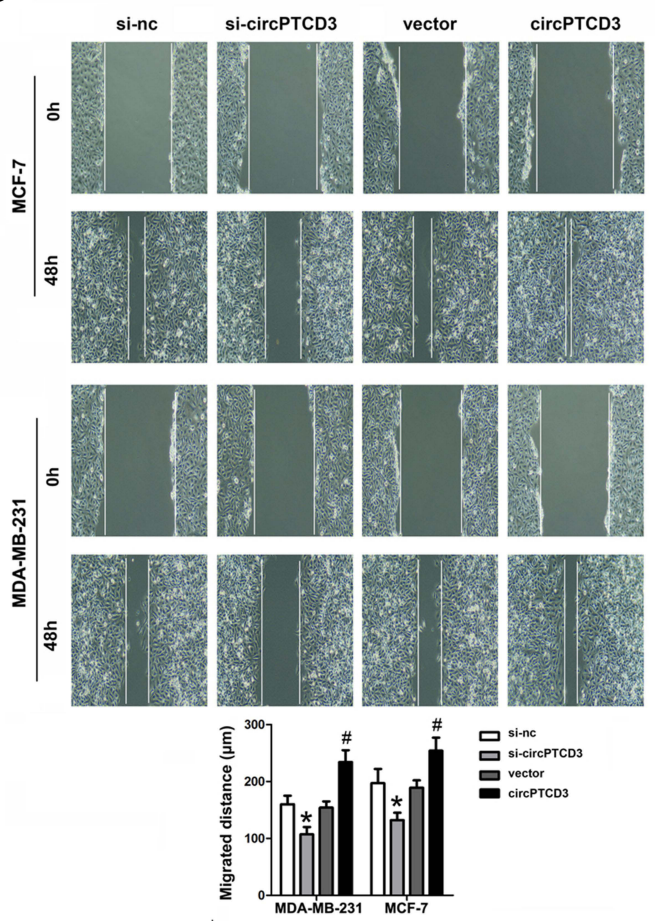

Figure 2 CircPTCD3 promoted the proliferation and migration of breast cancer cell. Breast cancer cells were transfected with overexpressing vector or siRNA for circPTCD3 as well as their negative control respectively. (A) qPCR was used to evaluate the expression level of circPTCD3. (B) MTT was performed to detect the proliferation of breast cancer cells in MCF-7 and MDA-MB-23I. (C) Wound healing assay was carried out to evaluate the migration of breast cancer cells. (D) Transwell assay was used to investigate the migration of the breast cancer cell. (E) Colony formation assay was performed to evaluate the colony formation ability of breast cancer cell. $n=6$, $* \mathrm{P}<0.05$, compared with the si-nc group. ${ }^{\#} \mathrm{P}<0.05$, compared with the vector group.

As the MTT results suggested, the circPTCD3 overexpression promoted cell proliferation while the circPTCD3 knock down inhibited that of breast cancer cells (Figure 2B). Furthermore, wound healing and transwell assay were carried out for the evaluation of the migration ability of breast cancer cells. As the results suggested, circPTCD3 overexpression reduced the migrated distance in the wound healing assay as well as the migrated cell number in transwell assay. On the contrary, the silencing of circPTCD3 lowered the capability of breast cancer cells as compared with the siRNA control group (Figure 2C and D). Colony formation was further carried out for the confirmation of our speculation. As expected, the circPTCD3 overexpression augmented the colony formation capability, while silencing of circPTCD3 lowered it in breast cancer cells (Figure 2E).

\section{CircPTCD3 Exerts Its Function by Sponging miR- 198}

It is well known that circRNAs sponge miRNAs for regulating the downstream gene expression. We carried out the bioinformatics analysis software, CircInteractome, aimed at predicting the potential miRNA target of circPTCD3, followed by selecting miR-198. Figure 3A demonstrates the complementary base sequences between circPTCD3 and miR-198. The luciferase activity assay was carried out for the verification of the binding of circPTCD3 and miR198. We discovered the fact that miR-198 lowered the luciferase activities of the wild-type reporter for circPTCD3 but not that of the mutant-type reporter, confirming miR-198 as a sponge target of circPTCD3 (Figure 3B). Subsequent to that, we found that circPTCD3 overexpression lowered the level of miR-198 while the circPTCD3 knock down increased that (Figure 3C). The RNA pull down was further carried out for the purpose of confirming the binding of circPTCD3 and miR-198. By means of the results, we figured it out that the enrichment of miR-198 was substantially higher in the circPTCD3 probe pull-down product in comparison with that of the control probe (Figure 3D). Accordingly, more circPTCD3 was captured by the biotinlabelled miR-198 groups as compared with the control probe (Figure 3E). Eventually, the Pearson analysis was carried out to determine the relationship between circPTCD3 and miR198. As the results suggested, there was a significant negative relationship existing between circPTCD3 and miR-198 

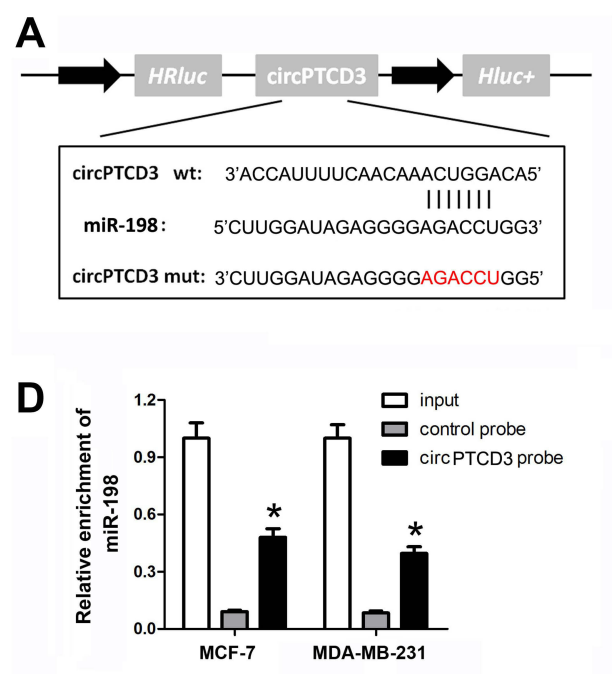

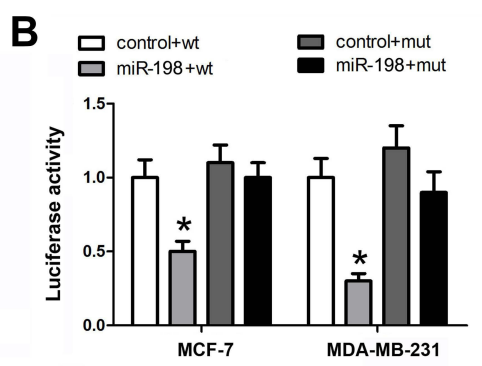

E

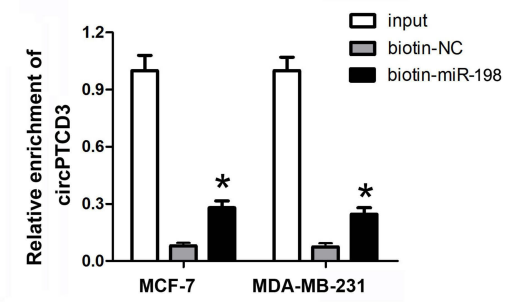

C
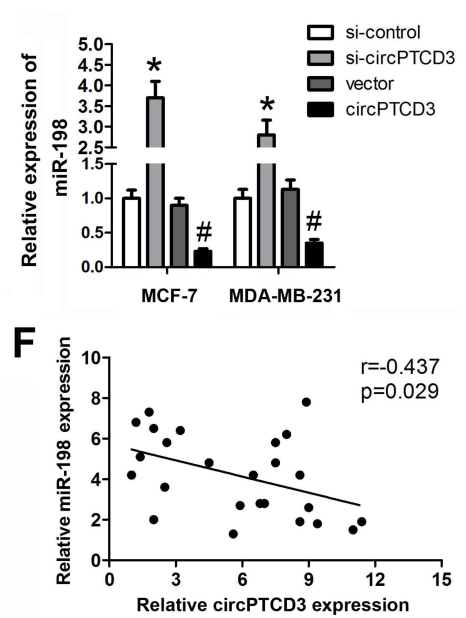

Figure 3 CircPTCD3 directly targets miR-198 in breast cancer cells. (A) The seed sequence of circPTCD3 that binds to miR-198 was showed, the sequences mutated were marked in red. (B) Luciferase reporter assay was carried out in breast cancer cell transfected with miR-198 mimic and the mimic control along with the reporter vector containing wild or mutant type of binding sequence of circPTCD3. (C) The level of miR-198 after different treatment was evaluated using qPCR. (D and E) RNA pull down was carried out using specific probe for circPTCD3 ormiR-198 respectively labeled by biotin. (F) Pearson analysis was performed to investigate the correlation between circPTCD3 and miR-198. $n=6,{ }^{*} P<0.05$, compared with the biotin-nc, control probe, si-nc and control+wt group. ${ }^{\#} \mathrm{P}<0.05$, compared with the vector group.

(Figure 3F). These findings suggested miR-198 as a sponge target of circPTCD3 in breast cancer cells.

\section{CircPTCD3 Reversed the Function of miR-198 in Breast Cancer Cells}

Being a target of circPTCD3, we predicted that miR-198 had involvement in the biological mechanism of breast cancer cells as well. We carried out the miR-198 mimic and circPTCD3 overexpressing vector transfection. The miR-198 mimic transfection apparently augmented the level of miR-
198; on the other hand, the co-transfection with miR-198 mimic and circPTCD3 overexpressing vector lowered the level of miR-198 in comparison with that in the miR-198 group (Figure 4A). By means of the function studies, we, at first, discovered that miR-198 attenuated not only the cell proliferation but also the migration and colony formation of breast cancer cells. Together with that, circPTCD3 reversed these functions of miR-198 partially, further indicating the relationship existing between circPTCD3 and miR-198 (Figure 4B-D).
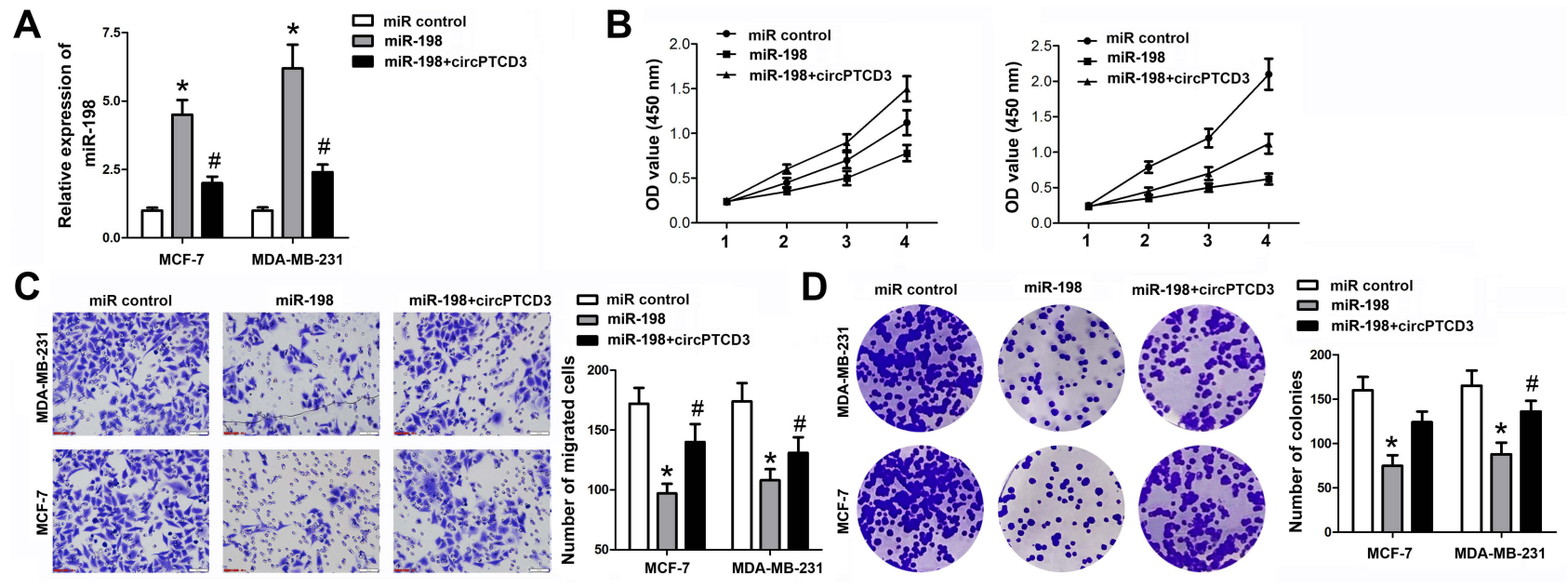

Figure 4 CircPTCD3 reversed the anticancer effect of miR-198 in breast cancer cell. (A) qPCR was used to detect the level of miR-198 in breast cancer cells under different treatment. (B) MTT was performed to evaluate the cell proliferation of breast cancer cells. (C) Transwell assay was used to detect the cell migration of breast cancer cells. (D) Colony formation assay was carried out to investigate the colony formation ability of breast cancer cells. $n=6,{ }^{*} \mathrm{P}<0.05$, compared with the normal group. ${ }^{\#} \mathrm{P}<0.05$, compared with the miR-198 group. 


\section{CircPTCD3 Promoted the Growth of Breast Cancer Cells in vivo}

Thereafter, we carried out the in vivo study to further investigate the biological function of circPTCD3 in the progression of breast cancer. Stable MCF-7 cell line was established with the transfection of sh-circPTCD3 or circPTCD3 vectors and their control, correspondingly. Both the size and weight of the tumours in the circPTCD3 were observed as higher in comparison with the control tumours. Contrarily, the circPTCD3 deletion lowered the tumour size and weight of tumours in comparison with the control group (Figure 5A-C). IHC assay, detecting the expression of ki67, the proliferation biomarker, was also carried out. In accordance with the expectations, circPTCD3 overexpression promoted the expression of ki67 while the knock-down of circPTCD3 inhibited that of ki67 (Figure 5D).

\section{Discussion}

CircRNAs have been fully documented as playing critical roles in the progression of breast cancer such as circRNF20, circular RNA Foxo3, circKDM4C, hsa_circ_001783, circDNMT1 and CircIRAK3. ${ }^{11-16}$ In the current study, we figured out an novel circRNA, termed as circPTCD3. We illustrated the fact that circPTCD3 originated from exons 3 to 9 of PTCD3, together with forming a loop structure by means of connecting the $3^{\prime}$ and $5^{\prime}$ splice sites. The stability of circPTCD3 was confirmed by its stable expression subjected to the RNase R digestion. To the best of our understanding, this is the first study dealing with the circPTCD3 emphasizing its modified expression as well as biological impact. The up-regulated expression and stability of circPTCD3 make it a potential biomarker as a diagnostic and therapeutic target.

Previous studies have demonstrated diverse mechanisms underlying the effect of circRNAs, such as acting as scaffolds of protein complexes, regulating protein subcellular localization, sequestering RNA-binding proteins, or functioning as the sponge of miRNAs. In the present study, we focus on the ability of circPTCD3 sponging miRNAs.

Plenty of miRs have been demonstrated as participating in the biological process of breast cancer. MiR-137, miR-17, miR-186-3p, miR-454-3p and miR-29a inhibited not only the proliferation but also the migration and invasion of human breast cancer cells by means of targeting the DUSP4, JAB1, EREG, Wnt $/ \beta$ and SUV420H2, correspondingly. ${ }^{17-20}$ Among them, miR-198, belonging to the miR-198 family, have been extensively investigated for playing a crucial role in the cellular differentiation and development. As in cancers, a number of research works shed light on the oncogenetic or anticancer function of miR-198, which include ovarian cancer, myeloid leukaemia, colorectal cancer, non-small cell
A

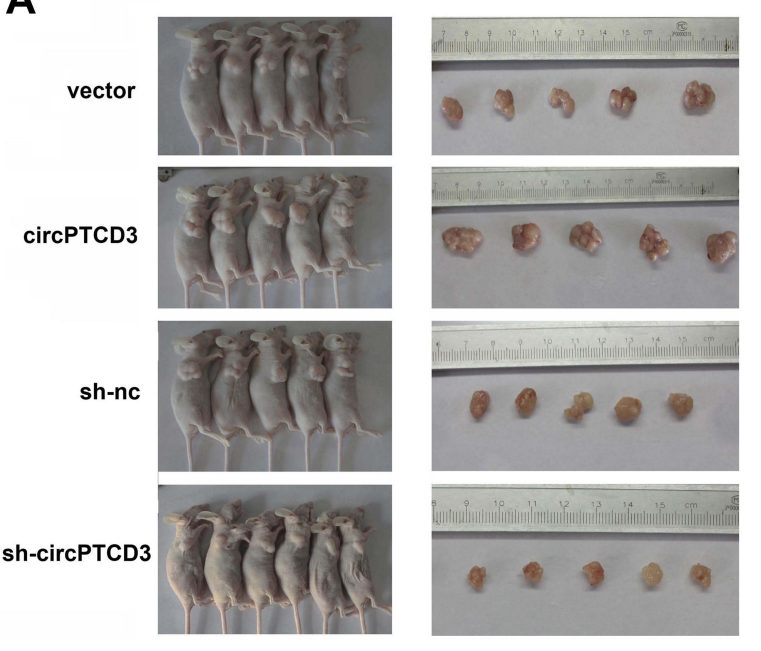

B

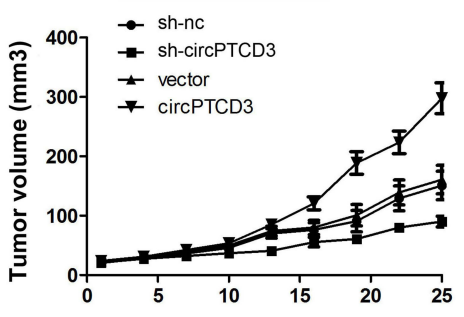

D

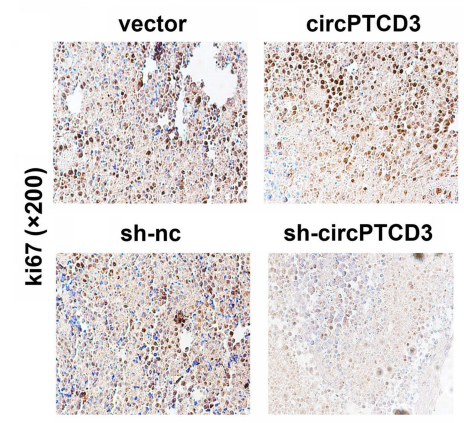

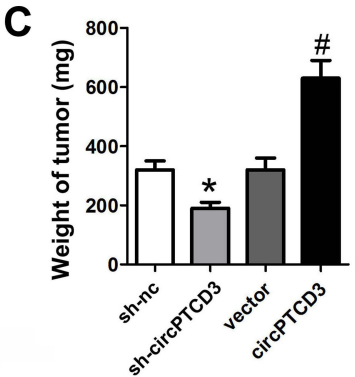

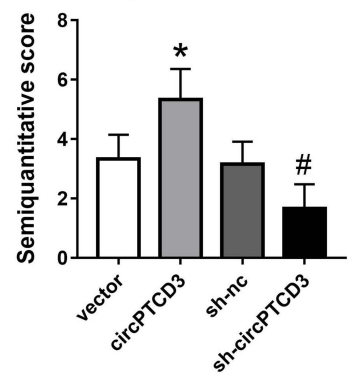

Figure 5 CircPTCD3 promoted the growth of breast cancer cells in vivo. Xenograft tumor model was established by subcutaneously injecting MCF-7 cells stably expressing sh-circPTCD3 or negative control in the dorsal flank area of nude mice. (A) The picture of the nude mice and the tumors in each group. (B) The growth curve of the tumor in the different groups. (C) The weight of the tumors in each group were calculated. (D) IHC was used to detect the ki67 expression $(\times 100)$. $n=5$, $* P<0.05$, compared with the biotin-nc, ${ }^{\#} \mathrm{P}<0.05$, compared with the vector group. 
lung cancer and gastric cancer. However, the role of miR-198 in the progression of breast cancer is limited. miR-198 has been demonstrated to targetLINC00473 and MAPK1 in breast cancer cells. $^{21}$ In addition to that, miR-198 was reported to act as an anticancer gene in breast cancer by targeting CUB domain-containing protein $1 .^{22}$ In the current research work, miR-198 was determined to be the target miRNA of circPTCD3, which was down-regulated in the breast cancer tissues and cells. Together with that, the function study revealed the anticancer effect of miR-198 in breast cancer, which is consistent with the previous study. Interestingly, miR-198 overexpression reversed the impact of circPTCD3, further verifying the relationship existing between them.

MiRNAs target mRNAs to induce the translation inhibition or degradation of them. ${ }^{23}$ The limitation of this study is that we have not figure out the downstream target gene of miR-198 in breast cancers. It will be carried out in the future.

To conclude, our findings shed light on the fact that circPTCD3 promotes the malignant behaviour of breast cancer by means of sponging miR-198, which indicates its potentiality to be a diagnostic or therapeutic target of breast cancer.

\section{Disclosure}

All authors declare that no conflicts of interest exist in the research.

\section{References}

1. Woolston C. Breast cancer. Nature. 2015;527(7578):S101. doi:10.1038/527S101a

2. Cook DJ, Kallus J, Jornsten R, Nielsen J. Molecular natural history of breast cancer: leveraging transcriptomics to predict breast cancer progression and aggressiveness. Cancer Med. 2020;9(10):3551-3562. doi:10.1002/cam4.2996

3. Khandekar D, Tiriveedhi V. Role of BET inhibitors in triple negative breast cancers. Cancers. 2020;12(4):784. doi:10.3390/ cancers 12040784

4. Zhang HD, Jiang LH, Sun DW, Hou JC, Ji ZL. CircRNA: a novel type of biomarker for cancer. Breast Cancer Tokyo. 2018;25(1):1-7. doi:10.1007/s12282-017-0793-9

5. Agostini M, Ganini C, Candi E, Melino G. The role of noncoding RNAs in epithelial cancer. Cell Death Discov. 2020;6(1):13. doi:10.1038/s41420-020-0247-6

6. Wei L, Sun J, Zhang N, et al. Noncoding RNAs in gastric cancer: implications for drug resistance. Mol Cancer. 2020;19(1):62. doi:10.1186/s12943-020-01185-7
7. Chen LL, Yang L. Regulation of circRNA biogenesis. RNA Biol. 2015;12(4):381-388. doi:10.1080/15476286.2015.1020271

8. Hua JT, Chen S, He HH. Landscape of noncoding RNA in prostate cancer. Trends Genet. 2019;35(11):840-851. doi:10.1016/j. tig.2019.08.004

9. Mohr AM, Mott JL. Overview of microRNA biology. Semin Liver Dis. 2015;35(1):3-11. doi:10.1055/s-0034-1397344

10. Lou W, Ding B, Fu P. Pseudogene-derived lncRNAs and their miRNA sponging mechanism in human cancer. Front Cell Dev Biol. 2020;8:85. doi:10.3389/fcell.2020.00085

11. Wu J, Jiang Z, Chen C, et al. CircIRAK3 sponges miR-3607 to facilitate breast cancer metastasis. Cancer Lett. 2018;430:179-192. doi:10.1016/j.canlet.2018.05.033

12. Du WW, Yang W, Li X, et al. A circular RNA circ-DNMT1 enhances breast cancer progression by activating autophagy. Oncogene. 2018;37(44):5829-5842. doi:10.1038/s41388-018-0369-y

13. Liu Z, Zhou Y, Liang G, et al. Circular RNA hsa_circ_001783 regulates breast cancer progression via sponging miR-200c-3p. Cell Death Dis. 2019;10(2):55. doi:10.1038/s41419-018-1287-1

14. Liang Y, Song X, Li Y, et al. circKDM4C suppresses tumor progression and attenuates doxorubicin resistance by regulating miR-548p/ PBLD axis in breast cancer. Oncogene. 2019;38(42):6850-6866. doi:10.1038/s41388-019-0926-z

15. Cao L, Wang M, Dong Y, et al. Circular RNA circRNF20 promotes breast cancer tumorigenesis and Warburg effect through miR-487a/ HIF-1alpha/HK2. Cell Death Dis. 2020;11(2):145. doi:10.1038/ s41419-020-2336-0

16. Huang W, Yang Y, Wu J, et al. Circular RNA cESRP1 sensitises small cell lung cancer cells to chemotherapy by sponging miR-93-5p to inhibit TGF-beta signalling. Cell Death Differ. 2019.

17. Min J, Han TS, Sohn Y, et al. microRNA-30a arbitrates intestinal-type early gastric carcinogenesis by directly targeting ITGA2. Gastric Cancer. 2020;23(4):600-613. doi:10.1007/s10120020-01052-w

18. He M, Jin Q, Chen C, et al. The miR-186-3p/EREG axis orchestrates tamoxifen resistance and aerobic glycolysis in breast cancer cells. Oncogene. 2019;38(28):5551-5565. doi:10.1038/s41388-019-0817-3

19. Ren L, Chen H, Song J, et al. MiR-454-3p-Mediated Wnt/beta-catenin Signaling Antagonists Suppression Promotes Breast Cancer Metastasis. Theranostics. 2019;9(2):449-465. doi:10.7150/ thno. 29055

20. Wu Y, Shi W, Tang T, et al. miR-29a contributes to breast cancer cells epithelial-mesenchymal transition, migration, and invasion via downregulating histone H4K20 trimethylation through directly targeting SUV420H2. Cell Death Dis. 2019;10(3):176. doi:10.1038/s41419019-1437-0

21. Niu L, Zhou Y, Zhang W, Ren Y. Long noncoding RNA LINC00473 functions as a competing endogenous RNA to regulate MAPK1 expression by sponging miR-198 in breast cancer. Pathol Res Pract. 2019;215(8):152470. doi:10.1016/j.prp.2019.152470

22. Hu Y, Tang Z, Jiang B, Chen J, Fu Z. miR-198 functions as a tumor suppressor in breast cancer by targeting CUB domain-containing protein 1. Oncol Lett. 2017;13(3):1753-1760. doi:10.3892/ ol.2017.5673

23. Caiment F, Gaj S, Claessen S, Kleinjans J. High-throughput data integration of RNA-miRNA-circRNA reveals novel insights into mechanisms of benzo[a]pyrene-induced carcinogenicity. Nucleic Acids Res. 2015;43(5):2525-2534. doi:10.1093/nar/gkv115 


\section{Publish your work in this journal}

Cancer Management and Research is an international, peer-reviewed open access journal focusing on cancer research and the optimal use of preventative and integrated treatment interventions to achieve improved outcomes, enhanced survival and quality of life for the cancer patient.

The manuscript management system is completely online and includes a very quick and fair peer-review system, which is all easy to use. Visit http://www.dovepress.com/testimonials.php to read real quotes from published authors.

Submit your manuscript here: https://www.dovepress.com/cancer-management-and-research-journal 